\title{
Interaction between Different Cells in Olfactory Bulb and Synchronous Kinematic Analysis
}

\author{
Ailei $\mathrm{Xu},{ }^{1,2}$ Ying Du, ${ }^{1,2}$ Rubin Wang, ${ }^{1,2}$ and Jinde $\mathrm{Cao}^{3,4}$ \\ ${ }^{1}$ School of Information Science and Engineering, East China University of Science and Technology, Shanghai 200237, China \\ ${ }^{2}$ School of Science, East China University of Science and Technology, Shanghai 200237, China \\ ${ }^{3}$ Department of Mathematics, Southeast University, Nanjing 210096, China \\ ${ }^{4}$ Department of Mathematics, Faculty of Science, King Abdulaziz University, Jeddah 21589, Saudi Arabia
}

Correspondence should be addressed to Rubin Wang; rbwang@163.com

Received 12 February 2014; Accepted 9 March 2014; Published 14 May 2014

Academic Editor: Guanghui Wen

Copyright (C) 2014 Ailei Xu et al. This is an open access article distributed under the Creative Commons Attribution License, which permits unrestricted use, distribution, and reproduction in any medium, provided the original work is properly cited.

\begin{abstract}
Olfactory bulb plays an important part in signal encoding of olfactory system. The interaction between excitatory mitral cell (MC) and inhibitory granule cell (GC) is particularly crucial. In this paper, the current situation of synchronous oscillation in the network of olfactory system is firstly introduced. Then we set up a dynamical model of MC and GC in the olfactory bulb. The simulation shows the firing patterns of single MC and single GC, as well as these two kinds of cells having a coupling relationship. The results indicate that MCs have an excitatory effect on GCs, and GCs have an inhibitory effect on MCs. The firing pattern varies with different synaptic strength. In addition, we set up simple olfactory network models, discussing the influence of ring-like and gridlike neuronal networks of GCs on the synchronization of two MCs. Different types of firing synchronization are quantified by means of ISI-distance method. The numerical analysis indicates that grid-like neuronal network can make MCs synchronize better.
\end{abstract}

\section{Introduction}

Olfactory system is an important part in sensory nervous system. Since there are many olfactory sensory neurons in our nose, we can sense the odour [1] and distinguish thousands of odours successfully. Each olfactory sensory neuron can express a specific odorant receptor. These receptors pass the odour information to olfactory bulb. Then the information will be the information will be transmitted to the entorhinal cortex of the brain by olfactory bulb, resulting in the sense of smell. As the first transfer station of the olfactory system, olfactory bulb plays an important role in processing olfactory information. When nerve signals coming from the depths of nasal get to the olfactory bulb, it will generate corresponding spatial and temporal coding [2]. The process of encoding information, in fact, is the process of further processing and handling information. Many scholars are discussing how the information processed by olfactory bulb is transferred synchronously to the entorhinal cortex.
In the olfactory bulb, there is an interaction between excitatory MCs and inhibitory GCs. The synaptic connections have several functions: (1) the lateral inhibition from GCs makes the receptive field of MCs smaller; (2) the interaction plays a role in oscillations formation in the olfactory bulb and also acts to synchronize the spikes of the MCs; (3) these synapses may be able to store the olfactory memory. Local field potential can be produced by the interaction between MCs and GCs [3]. The strength of synaptic connections determines the degree of the synchronization of the spikes, which is conducive to the integration of information. In this way, it can be more reliable to activate neurons of following olfactory cortex [4]. Thus there is a guarantee of transmitting signal and it lays a foundation for processing different olfactory information. Bazhenov et al. [5] and some other scholars put forward a network structural model of locust antenna, and then they verified the phenomenon of synchronous oscillation. On the concept of many-areequal (MAE), Brody and Hopfield [6] further checked that 
olfactory system can identify odour by the synchronization of spikes of MCs. When the odorousness is in a certain range, the synchronization of group cells is unchanged. Some scholars like Horcholle-Bossavit et al. [7] studied the encoding mechanism of locust olfactory system through spacetime coding model. The simulation results indicated that excitatory neurons were phase-locked in different oscillatory period and showed synchronization.

In conclusion, the synchronization of the spikes of MCs plays an important role in information encoding. The interaction between MCs and GCs in olfactory bulb is particularly critical. So it becomes necessary to analyse these two kinds of cells in detail. In this paper, we set up the dynamical model of MC and GC in the olfactory bulb. The simulation figures show the firing patterns of single MC and single GC, as well as these two kinds of cells having a coupling relationship. It shows that MCs can be synchronized by inhibition from GC, changing from irregular spiking to synchronous spiking. We wonder if the synchronization still exists when MCs are connected to different neuronal network of GCs and whether the degree of synchronization is different. To answer this question, we set up simple olfactory network models that consist of two MCs and nine GCs, discussing the influence of ring-like and grid-like neuronal network of GCs on the synchronization of two MCs.

\section{Dynamics Model of Olfactory System}

In this paper, MCs and GCs are implemented as conduction base models following Hodgkin-Huxley kinetics [8]. MCs are modeled using a single compartment while GCs are modeled using two compartments, representing a soma and dendrites. The membrane capacitance for all cells is $1 \mu \mathrm{F} / \mathrm{cm}^{2}$ and therefore not mentioned specifically in the voltage equations. In addition, the following units were used: conductance per unit area in $\mathrm{ms} / \mathrm{cm}^{2}$, current per unit area in $\mu \mathrm{A} / \mathrm{cm}^{2}$, and voltage in $\mathrm{mV}$ and time $(t)$ in $\mathrm{ms}$. The membrane potentials are calculated with the following kinetic equation:

$$
\frac{d v_{k}}{d t}=-I_{L}-\sum_{i} I_{i}-\sum_{s} I_{s}+\sum_{e} I_{e}+\sum_{j} g_{k, j}\left(v_{j}-v_{k}\right),
$$

where $I_{L}, I_{i}, I_{s}$, and $I_{e}$ are the leak, ionic, synaptic, and external currents, respectively. $g_{k, j}, v_{j}$, and $v_{k}$ represent the electronic conductance and voltages between adjacent compartments in a given cell.

There are two sodium currents $I_{\mathrm{Na}}$ and $I_{\mathrm{Na}_{p}}$ and three potassium currents $I_{\mathrm{K}_{\text {fast }}}, I_{\mathrm{K}_{A}}$, and $I_{\mathrm{K}_{s}}$, in MCs. The GC soma has a sodium current $I_{\mathrm{Na}}$ and three potassium currents $I_{\mathrm{K}}$, $I_{\mathrm{K}_{\mathrm{A}}}$, and $I_{\mathrm{K}_{M}}$. The GC dendrites have one sodium current $I_{\mathrm{Na}}$ and one potassium current $I_{\mathrm{K}}$. All the intrinsic currents are described by the following equation:

$$
I_{i}=g_{i} m^{M} h^{H}\left(v-E_{i}\right)
$$

where $g_{i}$ is the maximal conductance and $E_{i}$ the reversal potential. The activation and inactivation variables $m$ and $h$ raised to the power $M$ and $H$, respectively; follow the kinetic equation as follows:

$$
\begin{aligned}
& \frac{d m}{d t}=\frac{\left(m_{\infty}-m\right)}{\tau_{m}}, \\
& \frac{d h}{d t}=\frac{\left(h_{\infty}-h\right)}{\tau_{h}} .
\end{aligned}
$$

The parameters $m_{\infty}$ and $h_{\infty}$ and the time constants $\tau$ are not the same for different currents. Values for some parameters of MCs and GCs are given in Table $1[8,9]$.

For the current $I_{\mathrm{K}_{\text {fast }}}$ in the MC and the currents $I_{\mathrm{Na}}$ and $I_{\mathrm{K}}$ in the GC dendrites and soma, we used values as shown in Figure 1 [9].

Synaptic connections between neurons are in the form of channel connectivity [10]. When MCs excite GCs, GCs will receive excitatory effect coming from receptors like APMA and NMDA. Accordingly, MCs will receive inhibitory effect from receptor GABA when inhibited by GCs. The strength of synaptic connections between cells is expressed by excitatory and inhibitory strength. The synaptic equations and relevant parameters are described in Table 2 [8].

\section{Simulation Results and Analysis}

3.1. Simulation of the Action Potential. Figures 2 and 3 are the spike trains of a single MC and GC under different external currents, respectively. Figure 2(a) is the membrane potential of $\mathrm{MC}$ without any external stimulus. When the stimulus gradually increases to $2 \mathrm{nA}$ and $4 \mathrm{nA}$, the firing frequency increases at the same time. For GC, the spiking frequency also increases when stimulus varies from $0 \mathrm{nA}$ to $10 \mathrm{nA}$ and $20 \mathrm{nA}$. It shows that the response of cell changes with different stimulus, consistent with the sensitivity to outside stimuli of the olfactory system.

We can find that the firing frequency of MC has a great change when the stimulus only increases by $2 \mathrm{nA}$. While for $\mathrm{GC}$, the spiking frequency just changes a little with stimulus adding $10 \mathrm{nA}$. By comparing these two group figures, maybe we can conclude that the firing patterns of MC change more obviously than GC with different stimulus.

3.2. Interaction between $M C$ and GC. In the olfactory bulb, $\mathrm{MC}$ has an excitatory effect on GC, while GC has an inhibitory effect on MC. Cells coupled by synaptic connections and the firing patterns change according to different connection strength. We choose to change GABA conductance as an example to investigate the firing patterns of MC under different inhibition strength. In addition, we also discussed how the spike trains of MC varied when coupled with a single GC.

As is shown in Figures 4 and 5, the firing frequency of GC increases when it gets an excitatory effect from MC. And when MC gets an inhibitory effect from GC, the spiking frequency decreases. This phenomenon becomes 


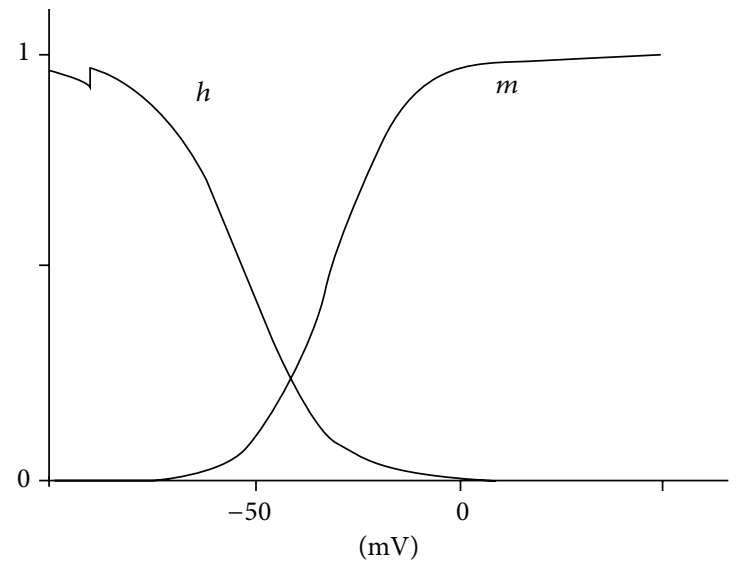

(a)

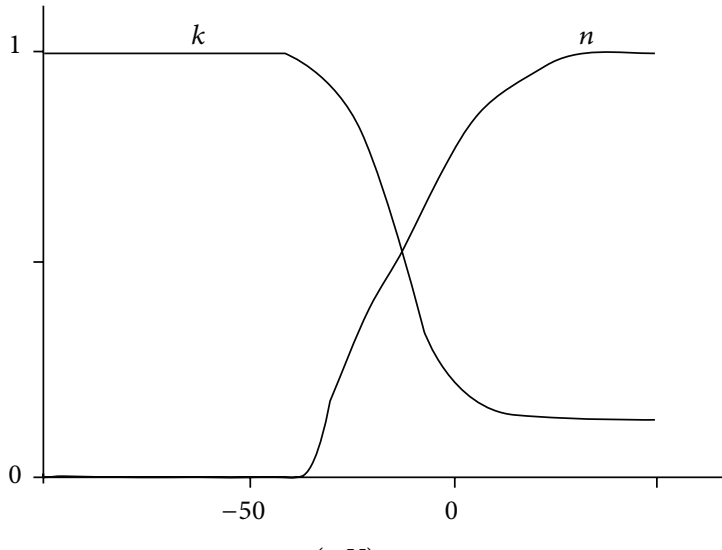

(mV)

(c)

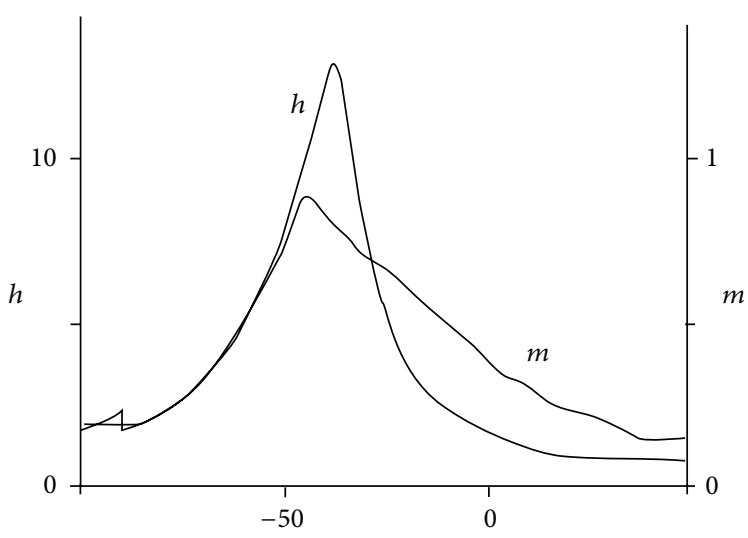

$(\mathrm{mV})$

(b)

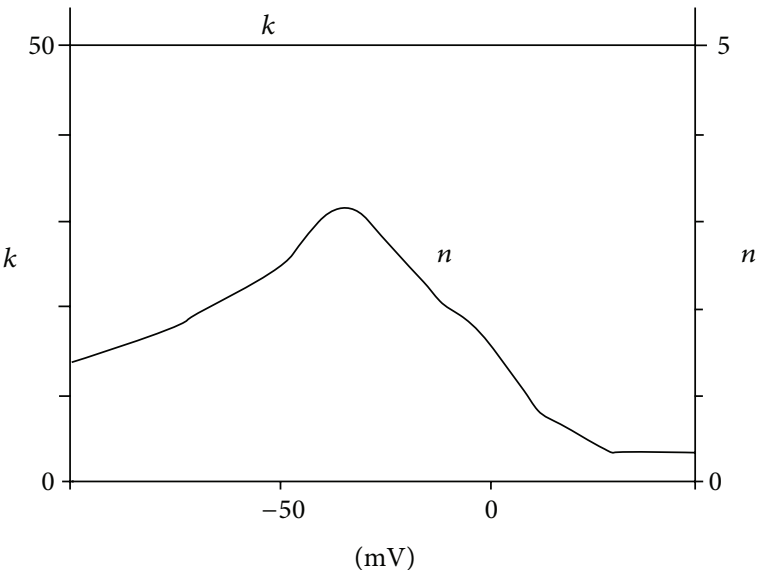

(d)

Figure 1: (a) $m_{\infty}$ and $h_{\infty}$ curves for Na channel of GC; (b) $\tau$ curves for Na channel of GC; (c) $m_{\infty}$ and $h_{\infty}$ curves for K and $\mathrm{K}_{\text {fast }}$ channels; (d) $\tau$ curves for $\mathrm{K}$ and $\mathrm{K}_{\text {fast }}$ channels. The $\tau$ values for $\mathrm{K}$ channel are scaled up by a factor of 4 from the graph.

TABLE 1: Some parameters in the dynamical model.

\begin{tabular}{|c|c|}
\hline \multicolumn{2}{|r|}{ Mitral cells } \\
\hline Na ion channel & $\begin{array}{l}M=3, H=1, E_{\mathrm{Na}}=45, a_{m}=0.32(v+50) /\{1-\exp [-(v+50) / 4]\}, b_{m}=0.28(v+23) /\{\exp [(v+23) / 5]-1\} \\
a_{h}=0.128 / \exp [(v+46) / 18], b_{h}=4 /\{1+\exp [-(v+23) / 5]\}, m_{\infty}=a_{m} /\left(a_{m}+b_{m}\right), h_{\infty}=a_{h} /\left(a_{h}+b_{h}\right), \\
\tau_{m}=1 /\left(a_{m}+b_{m}\right), \tau_{h}=1 /\left(a_{h}+b_{h}\right)\end{array}$ \\
\hline $\mathrm{K}_{\mathrm{s}}$ ion channel & $\begin{array}{l}M=1, H=1, E_{K_{s}}=-70, h_{\infty}=1 /\{\exp [(v+65) / 6.6]+1\}, \tau_{m}=10, m_{\infty}=1 /\{\exp (-[v+34) / 6.5]+1\} \\
\tau_{h}=2000+220 /\{\exp [-(v+71.6) / 6.85]+1\}\end{array}$ \\
\hline $\mathrm{K}_{\mathrm{A}}$ ion channel & $\begin{array}{l}M=1, H=1, E_{\mathrm{K}_{A}}=-70, m_{\infty}=1 /\{\exp [-(v-70) / 14]+1\}, h_{\infty}=1 /\{\exp [(v+47.4) / 6]+1\} \\
\tau_{m}=25 \exp [(v+45) / 13.3] /\{\exp [(v+45) / 10]+1\}, \tau_{h}=\cdot 55.5 \exp [(v+70) / 5.1] /\{\exp [(v+70) / 5]+1\}\end{array}$ \\
\hline $\mathrm{Na}_{P}$ ion channel & $I_{\mathrm{Na}_{p}}=g \bullet_{\mathrm{Na}_{p}}(1 /(\exp (-(v+51) / 5)+1))(v-45)$ \\
\hline \multicolumn{2}{|r|}{ Granule cell soma } \\
\hline $\mathrm{K}_{\mathrm{A}}$ ion channel & $M=1, H=1, E_{\mathrm{K}_{A}}=-70, m_{\infty}=1 /(1+\exp (-(v+42) / 13)), h_{\infty}=1 /(1+\exp ((v+110) / 18)), \tau_{m}=1.38, \tau_{h}=150$ \\
\hline $\mathrm{K}_{\mathrm{M}}$ ion channel & $M=3, H=0, E_{\mathrm{K}_{M}}=-70, m_{\infty}=1 /(\exp (-(v+35) / 5)+1), \tau_{m}=1000 /(3.3 \exp ((v+35) / 40)+\exp (-(v+35) / 20))$ \\
\hline
\end{tabular}

TABLE 2: Equations and some parameters of synaptic currents.

\begin{tabular}{ll}
\hline & \multicolumn{1}{c}{ Synaptic currents } \\
\hline GABA channel & $i_{\mathrm{GABA}}=g_{\mathrm{GABA}}\left(\exp \left(-t / \tau_{2}\right)-\exp \left(-t / \tau_{1}\right)\right)\left(v-e_{\mathrm{GABA}}\right), \tau_{1}=1, \tau_{2}=200, e_{\mathrm{GABA}}=-80$ \\
\hline APMA channel & $i_{\mathrm{AMPA}}=g_{\mathrm{AMPA}} \exp (-t / \tau)\left(v-e_{\mathrm{AMPA}}\right), \tau=3, e_{\mathrm{AMPA}}=0$ \\
\hline NMDA channel & $\begin{array}{l}i_{\mathrm{NMDA}}=\operatorname{mg}(\operatorname{Ron}+\operatorname{Roff})\left(v-e_{\mathrm{NMDA}}\right), \operatorname{Ron}^{\prime}=-\operatorname{Ron} / 2.6, \mathrm{mg}=1 /(1+\exp (-0.062 v) / 3.57), \operatorname{Roff}^{\prime}=-0.035 \operatorname{Roff}, \\
e_{\mathrm{NMDA}}=0\end{array}$ \\
\hline
\end{tabular}




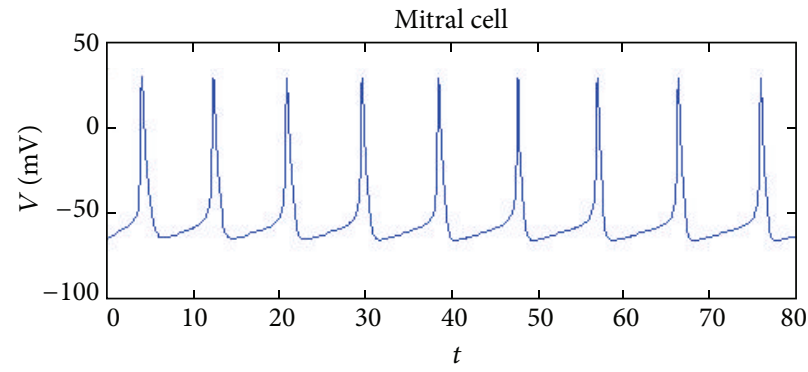

Membrane potential of single $\mathrm{MC}$ when $I_{e}=0 \mathrm{nA}$

(a)

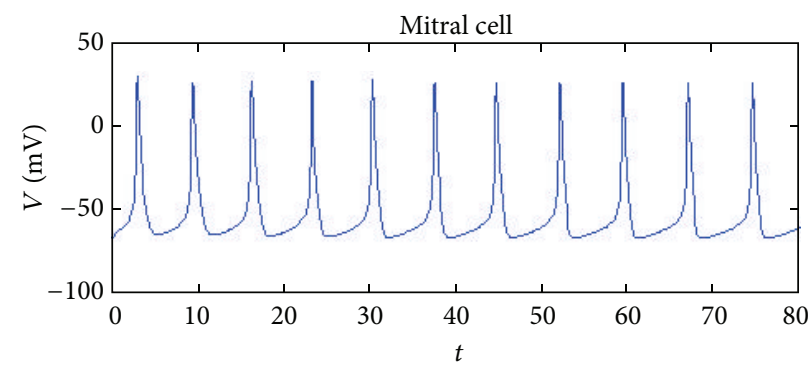

Membrane potential of single MC when $I_{e}=2 \mathrm{nA}$

(b)

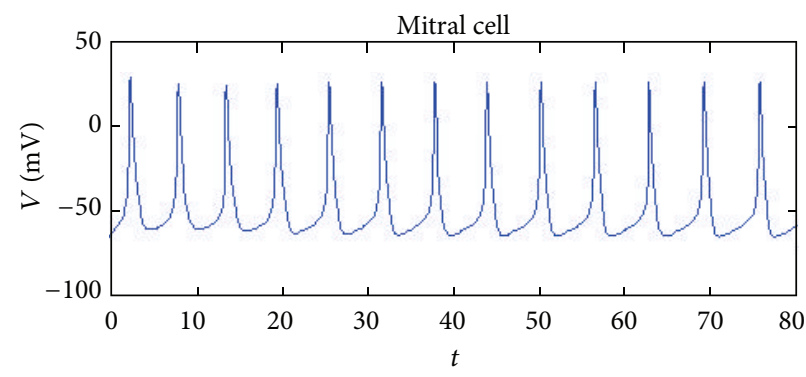

Membrane potential of single MC when $I_{e}=4 \mathrm{nA}$

(c)

Figure 2: Spike trains of a MC under different stimulus.

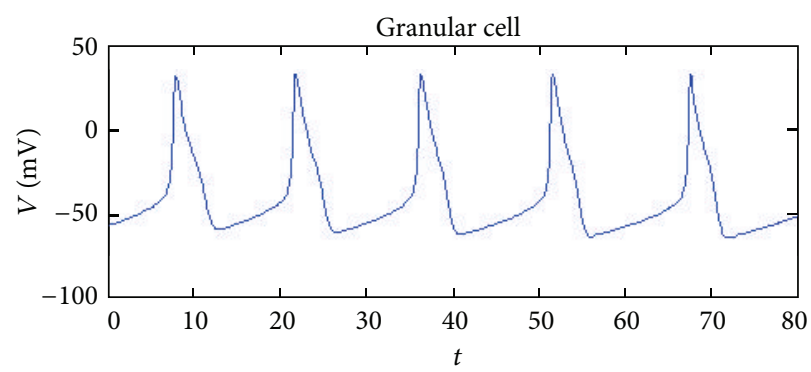

Membrane potential of single GC when $I_{e}=0 \mathrm{nA}$

(a)

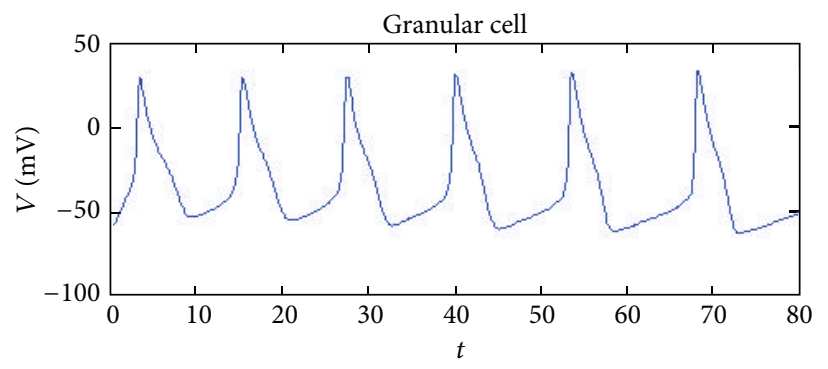

Membrane potential of single GC when $I_{e}=10 \mathrm{nA}$

(b)

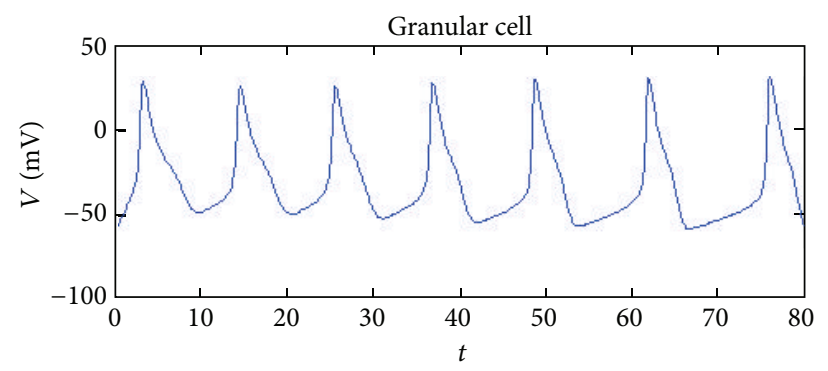

Membrane potential of single GC when $I_{e}=20 \mathrm{nA}$

(c)

FIGURE 3: Spike trains of a GC under different stimulus. 


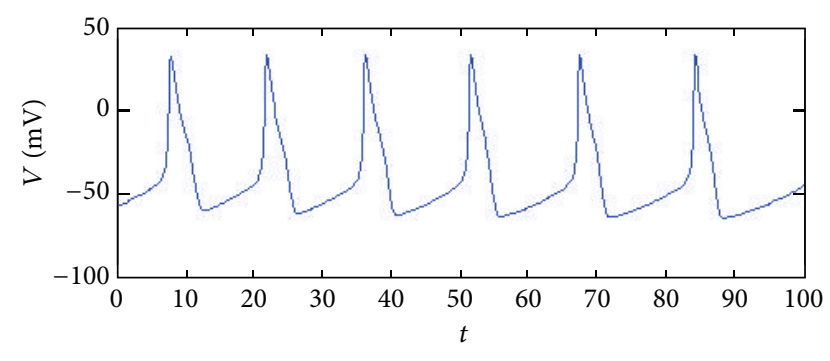

FIGURE 4: Membrane potential of a GC when $g_{\mathrm{AMPA}}=0$.

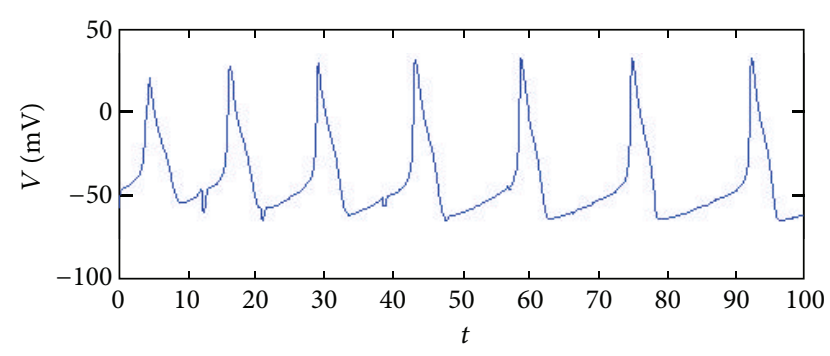

FIGURE 5: Membrane potential of a GC when $g_{\mathrm{AMPA}}=0.1$.

more obvious as the inhibitory strength increases, as shown in Figures 6, 7, 8, and 9.

(1) MC Having Excitatory on GC. See Figures 4 and 5.

(2) GC Having an Inhibitory Effect on MC. See Figures 6, 7, 8, and 9.

(3) MCs Spike Synchronously When Coupled with GC. The response of five MCs to stimulus without synaptic connections is shown in Figure 10(a). MC population spike asynchronously, for each MC getting different stimulus. When MCs got an inhibitory effect from GC, the firing rate of MCs decreases and they spike synchronously, as shown in Figure 11(a).

Figures 10(b) and 11(b) are the corresponding LFP plots of Figures 10(a) and 11(a). LFP reflects the excitement of local network, aiming to analyse the collaborative behavior of MC population. LFP focuses on the collaborative function between cells, hiding the individual role of each neuron. It is of great importance to discuss synchronous oscillation in the olfactory network. The LFP is defined as th mean value of the membrane potential of all MCs. When MC population spikes asynchronously, the LFP shows high frequency and low amplitude. While MC population fires synchronously, the LFP shows low frequency and high amplitude.

With the increase in the number of MCs, the curve of different membrane potential will be confused. Therefore, when the number reaches 20 , we take the raster plot to characterize the spiking activity of cell population. Figure $12(\mathrm{a})$ is the raster plot of MCs firing irregularly with no synaptic connections. With an inhibition from GC, MCs spike regularly with a slow speed, as shown in Figure 13(a). Figures 12(b) and 13(b) are the corresponding LFP plot of Figures 12(a) and 13(a).

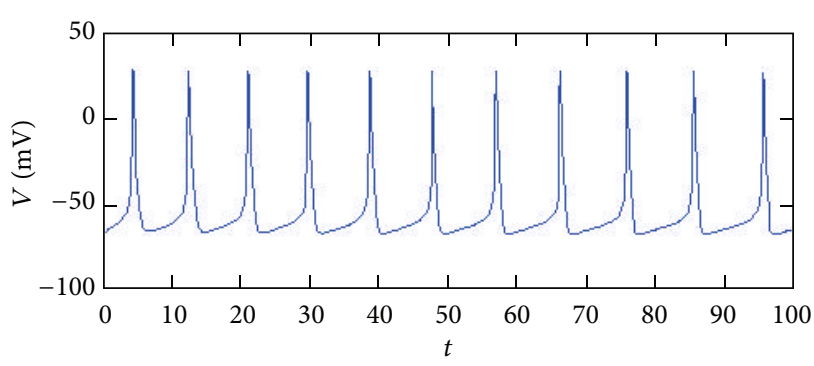

FIGURE 6: Membrane potential of a MC when $g_{\mathrm{GABA}}=0$.

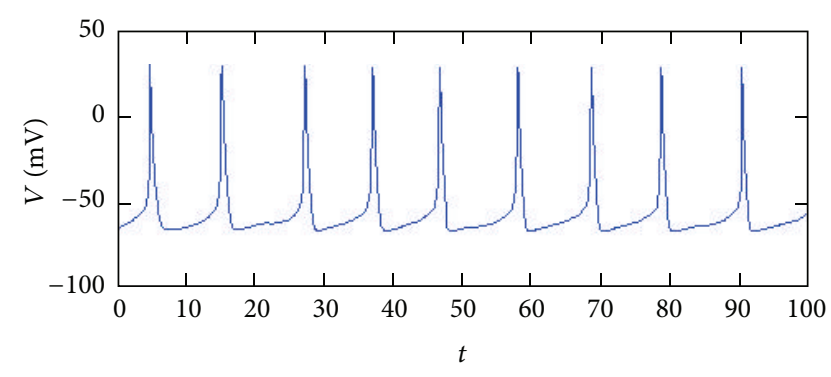

FIGURE 7: Membrane potential of a MC when $g_{\mathrm{GABA}}=0.03$.

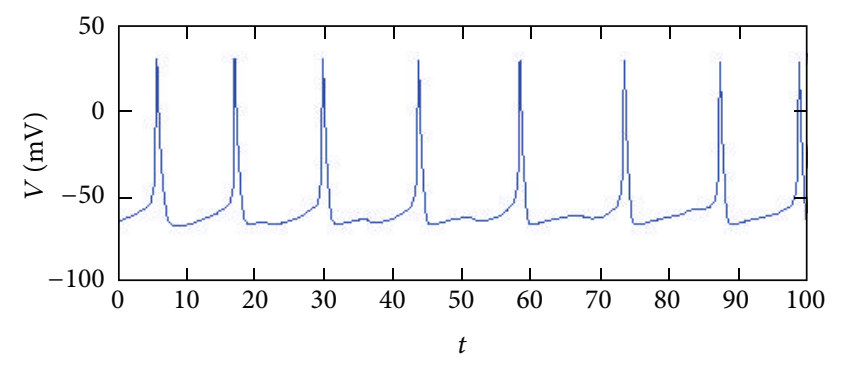

FIGURE 8: Membrane potential of a MC when $g_{\mathrm{GABA}}=0.06$.

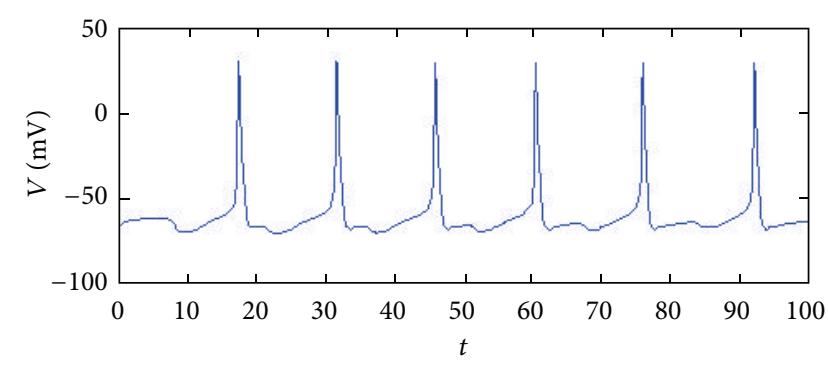

FIGURE 9: Membrane potential of a MC when $g_{\mathrm{GABA}}=0.1$.

3.3. Network Model of Olfactory Bulb. Based on the previous work, we found that MCs spiked irregularly before; now they can spike synchronously with inhibition from a single GC. We wonder if the synchronization still exists when MCs connected to different neuronal network of GCs and whether the degree of synchronization is different.

To figure out this question, we set up simple olfactory network models consisting of two MCs, nine GCs, and several synaptic connections. We bring in GABA receptor in GC-MC 


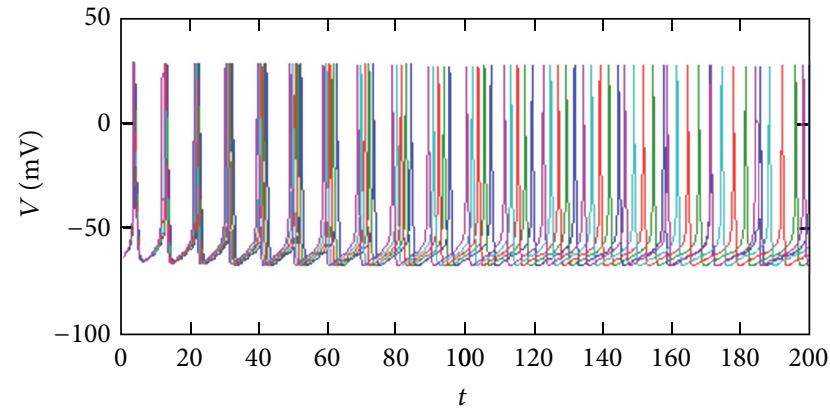

(a)

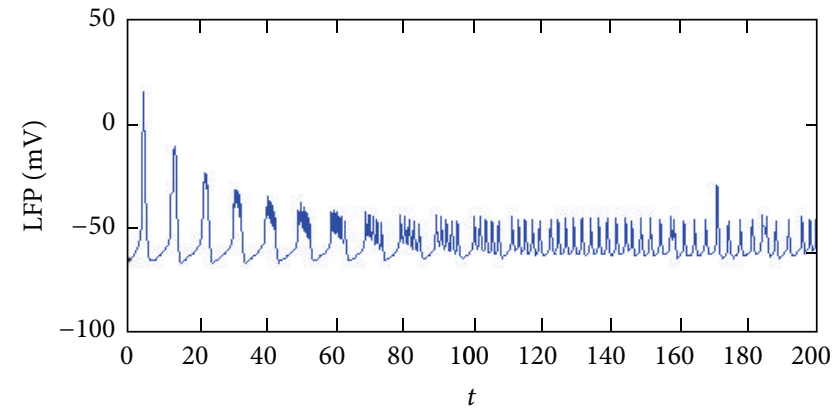

(b)

FIGURE 10: (a) Membrane potential of five MCs when $g_{\mathrm{GABA}}=0$. (b) LFP of five MCs when $g_{\mathrm{GABA}}=0$.

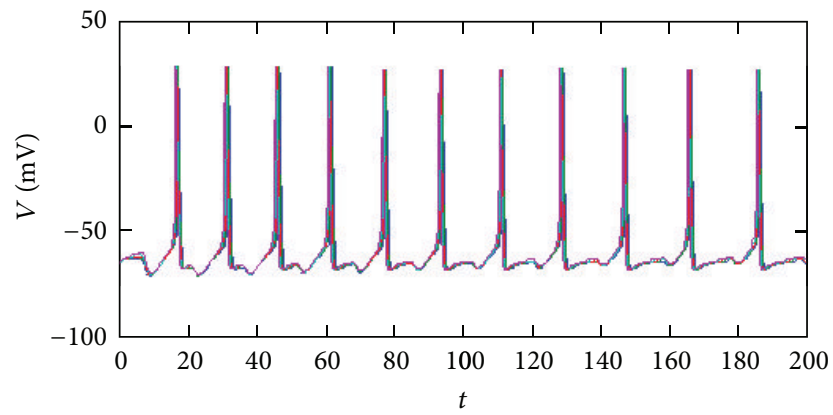

(a)

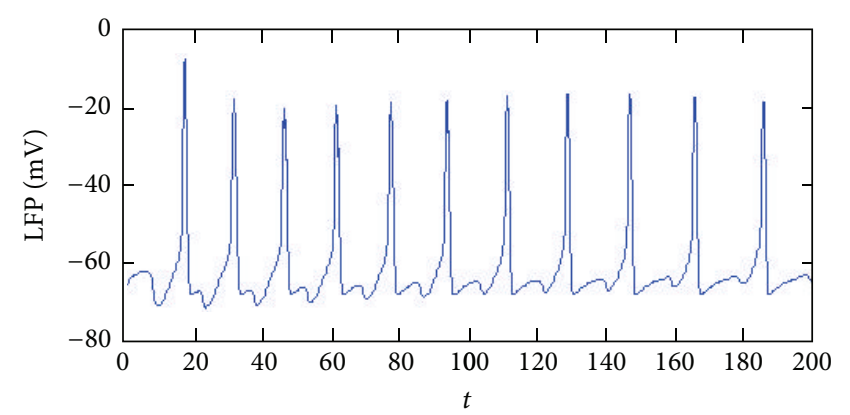

(b)

FIGURE 11: (a) Membrane potential of five MCs when $g_{\mathrm{GABA}}=0.1$. (b) LFP of five MCs when $g_{\mathrm{GABA}}=0.1$.

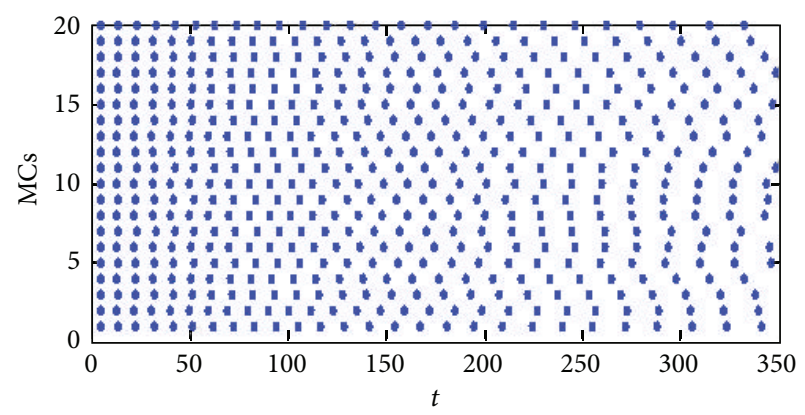

(a)

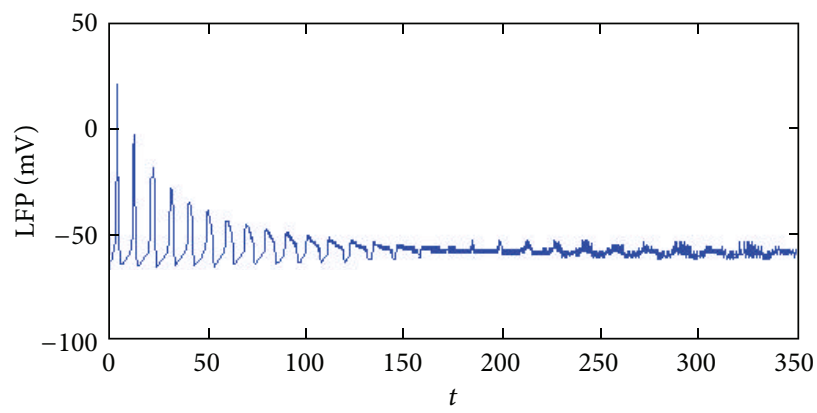

(b)

FIGURE 12: (a) Raster pattern of twenty MCs when $g_{\mathrm{GABA}}=0$. (b) LFP of twenty MCs when $g_{\mathrm{GABA}}=0$.

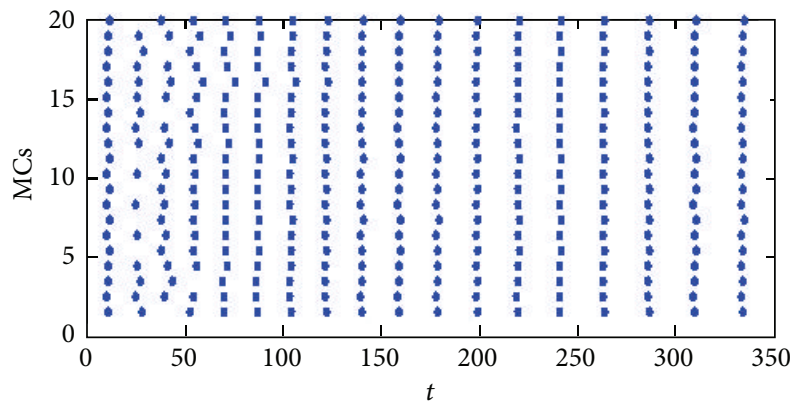

(a)

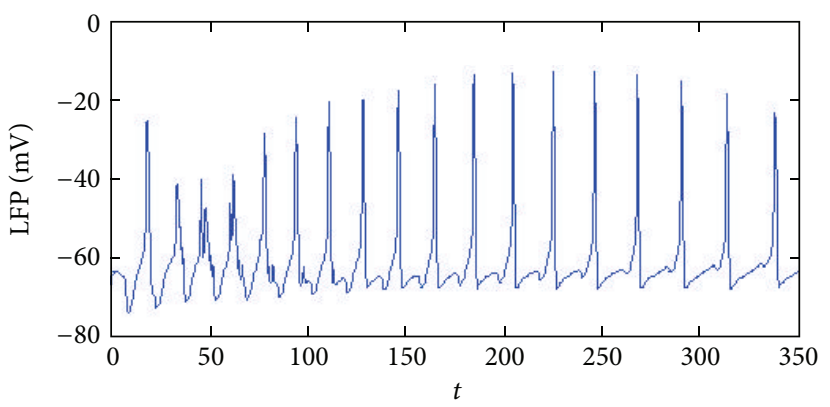

(b)

FIGURE 13: (a) Raster pattern of twenty MCs when $g_{\mathrm{GABA}}=0.1$. (b) LFP of twenty MCs when $g_{\mathrm{GABA}}=0.1$. 


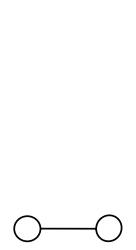

(a)

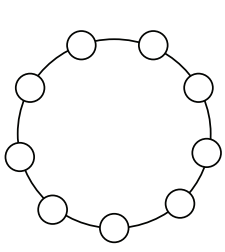

(b)

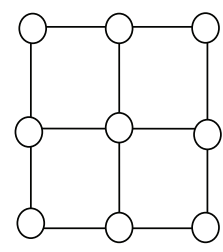

(c)
FIGURE 14: (a) Two coupled MCs, (b) ring-like neuronal network of GCs, and (c) grid-like neuronal network of GCs.

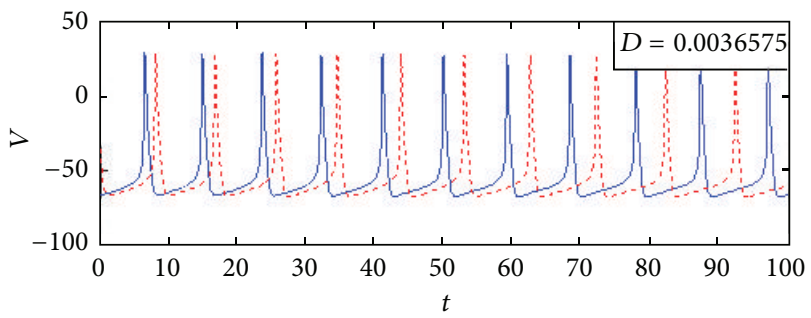

FIGURE 15: Membrane potential of two coupled MCs when $g_{\text {GABA }}=$ 0 .

and GC-GC synapses and also bring in AMPA and NMDA receptor in MC-GC synapses. We discussed how the ringlike and grid-like neuronal network of GCs influenced the synchronization of two MCs, respectively [11, 12].

In this model, the connection between MCs and two different topologies of GCs are shown in Figure 14. The ring-like neuronal network of GC only considers nearestneighbour neurons while the grid-like neuronal network is with all nearest neighbour couplings. We have already known that coupled MCs could achieve synchronization with the variation of coupling strength without inhibition from GCs [13]. In order to exclude the interference, we take the coupling strength of MCs as 0.01 . In this way, there is weak coupling relationship between two MCs.

In the present paper, the synchronization degree of two spike trains is quantified by the ISI-distance [14], which is suitable for both two and multiple neurons. It uses the interspike interval (ISI) instead of spike frequency as the basic element of comparison. The method is a simple complementary approach that extracts information from the interspike intervals by evaluating the ratio of the instantaneous firing rates. It is parameter-free, time scale independent, and easy to visualize. Firstly, we take the instantaneous ISI-ratio between two interspike intervals, $x_{\mathrm{ISI}}^{1}$ and $x_{\mathrm{ISI}}^{2}$, and then normalize it. When two spike trains are the same, the quantity of ISI-ratio $I_{1,2}(t)$ becomes zero. While if the first spike train is much higher or lower than the second spike train, it approaches -1 and 1 , respectively. In order to derive a measure of spike train distance, the absolute ISI-distance $D$ is integrated over time. The more synchronous the coupled neurons are, the less the ISI-distance $D$ is, and the complete synchronization state of

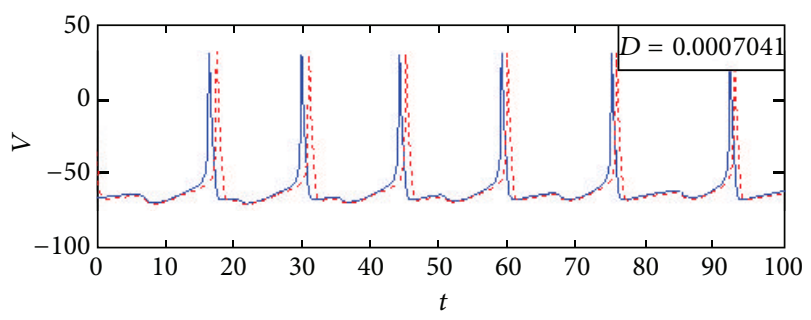

FIgURE 16: Membrane potential of two coupled MCs when connected to ring-like neuronal network of GCs.

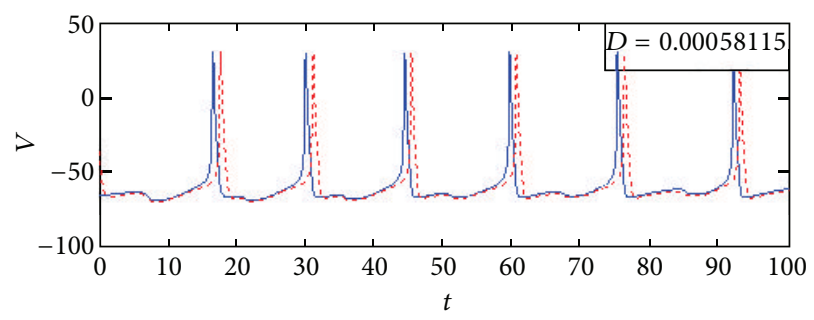

FIGURE 17: Membrane potential of two coupled MCs when connected to grid-like neuronal network of GCs.

the coupled neurons is achieved when $D$ is equal to 0 . The calculation formula is as follows:

$$
I_{1,2}(t)= \begin{cases}\frac{x_{\mathrm{ISI}}^{1}(t)}{x_{\mathrm{ISI}}^{2}(t)}-1 & x_{\mathrm{ISI}}^{1}(t) \leq x_{\mathrm{ISI}}^{2}(t) \\ -\left(\frac{x_{\mathrm{ISI}}^{2}(t)}{x_{\mathrm{ISI}}^{1}(t)}-1\right) & x_{\mathrm{ISI}}^{1}(t)>x_{\mathrm{ISI}}^{2}(t), \\ D=\int_{t=0}^{T} d t|I(t)| .\end{cases}
$$

The membrane potential curve of two MCs without inhibition from GCs networks is shown in Figure 15. When coupled with ring-like and grid-like neuronal networks of GCs, the membrane potential plots of MCs have some change, as illustrated in Figures 16 and 17, respectively.

As shown in Figure 15, the solid line and dotted line are the spike trains of two MCs without connection with GCs network, respectively. These two MCs spike in different moment, and $D$ is equal to $3.6575 \times 10^{-3}$. Compared with Figure 15, the spiking frequency of MCs decreases when coupled with ring-like network of GCs. At the same time, we can find that there is an apparent synchronization between two spike trains and $D$ is equal to $0.7041 \times 10^{-3}$. When coupled with grid-like network, the response is similar to Figure 16. Two MCs spike synchronously in a low speed, and $D$ is equal to $0.5811 \times 10^{-3}$ at the time. Comparing Figure 15 with Figures 16 and 17, it shows that MCs spike irregularly without connection with GCs, turning to regular spiking slowly when coupled with GCs networks.

Comparing Figures 16 and 17, we can find similar synchronous spike trains of MCs when coupled with different topology network of GCs. MCs fire synchronously, but the 
synchronization degree is a little different. According to ISIdistance $D$, it shows that MCs become more synchronous when coupled with grid-like neuronal network of GCs.

\section{Conclusion}

In this paper, we have done some simulation work based on the dynamical model of MC and GC in the olfactory bulb. The simulation results show the firing patterns of a single $\mathrm{MC}$ and a single GC, as well as these two kinds of cells having a coupling relationship. In addition, we set up simple olfactory network models, discussing the influence of ring-like and grid-like neuronal networks of GCs on the synchronization of two MCs. ISI-distance method has been used to measure the synchronization degree of MCs. In summary, we have got four outcomes.

(1) The spike trains of MC and GC change with different external currents. When there is a larger external current, the spiking frequency of neuron increases accordingly. By comparison, we may conclude that the firing patterns of MC change more obviously than GC with different stimulus.

(2) MC has an excitatory effect on GC. While GC has an inhibitory effect on MC. The firing frequency of GC increases when it gets an excitatory effect from MC. And when MC gets an inhibitory effect from GC, the spiking frequency decreases. Cells coupled by synaptic connections and the firing patterns change accordingly with different connection strength.

(3) MCs spike asynchronously when there is no connection with GC, and LFP shows high frequency and low amplitude. When MCs got an inhibitory effect from GC, the firing rate of MCs decreases and they spike synchronously at the same time. The LFP shows low frequency and high amplitude.

(4) In the simple olfactory network models, we discussed how the ring-like and grid-like neuronal networks of GCs influenced the synchronization of two MCs, respectively. Through numerical analysis, it shows that MCs spike irregularly without connection with GCs, turning to regular spiking slowly when coupled with GCs networks. Compared with ring-like network, MCs become more synchronous when coupled with grid-like neuronal network of GCs.

Since the synchronization of the spikes of MCs plays an important role in information encoding and the interaction between MCs and GCs in olfactory bulb is particularly critical, we have made a detailed analysis on excited MC and inhibitory GC. These results are important both for understanding the sense of smell and for understanding the mechanisms of neural computation. The study of the neural basis of olfactory system lays a foundation for our later work about analysing how the neurons in olfactory bulb play a role in information coding comprehensively and quantificationally, starting from the overall structure of olfactory bulb. It can also help us study the odour pattern recognition based on olfactory bulb physiological structure. In this paper, our research about olfactory cognitive function is below the entorhinal cortex. In the future work, we will discuss the formation mechanism of smell more roundly, combining the information processing process of entorhinal cortex.

\section{Conflict of Interests}

The authors declare that there is no conflict of interests regarding the publication of this paper.

\section{Acknowledgments}

This work was supported by the National Natural Science Foundation of China under (Grant nos. 11232005, 11002055, and 11172086) and The Ministry of Education Doctoral Foundation (20120074110020).

\section{References}

[1] J. Tan and M. Luo, "Olfactory information processing by the olfactory bulb," Journal of Biological Physics, vol. 26, no. 3, pp. 194-208, 2010.

[2] S. Wolf, "Distributed processing and temporal codes in neuronal networks," Cognitive Neurodynamics, vol. 3, no. 3, pp. 189196, 2009.

[3] Q. Zhen, "New development of olfactory recognition model," Journal of Wenzhou Medical College, vol. 39, no. 1, 2009.

[4] R. Wang, Z. Zhang, J. Qu, and J. Cao, "Phase synchronization motion and neural coding in dynamic transmission of neural information," IEEE Transactions on Neural Networks, vol. 22, no. 7, pp. 1097-1106, 2011.

[5] M. Bazhenov, M. Stopfer, M. Rabinovich et al., "Model of transient oscillatory synchronization in the locust antennal lobe," Neuron, vol. 30, no. 2, pp. 553-567, 2001.

[6] C. D. Brody and J. J. Hopfield, "Simple networks for spiketiming-based computation, with application to olfactory processing," Neuron, vol. 37, no. 5, pp. 843-852, 2003.

[7] G. Horcholle-Bossavit, B. Quenet, and O. Foucart, "Oscillation and coding in a formal neural network considered as a guide for plausible simulations of the insect olfactory system," BioSystems, vol. 89, no. 1-3, pp. 244-256, 2007.

[8] J. N. Brea, L. M. Kay, and N. J. Kopell, "Biophysical model for gamma rhythms in the olfactory bulb via subthreshold oscillations," Proceedings of the National Academy of Sciences of the United States of America, vol. 106, no. 51, pp. 21954-21959, 2009.

[9] U. S. Bhalla and J. M. Bower, "Exploring parameter space in detailed single neuron models: simulations of the mitral and granule cells of the olfactory bulb," Journal of Neurophysiology, vol. 69, no. 6, pp. 1948-1965, 1993.

[10] D. Friedman and B. W. Strowbridge, "Both electrical and chemical synapses mediate fast network oscillations in the olfactory bulb," Journal of Neurophysiology, vol. 89, no. 5, pp. 2601-2610, 2003.

[11] J. Qu, R. Wang, Y. Du, and J. Cao, "Synchronization study in ring-like and grid-like neuronal networks," Cognitive Neurodynamics, vol. 6, no. 1, pp. 21-31, 2012. 
[12] Y. Liu, R. Wang, Z. Zhang, and X. Jiao, "Analysis of stability of neural network with inhibitory neurons," Cognitive Neurodynamics, vol. 4, no. 1, pp. 61-68, 2010.

[13] Y. Du, R. Wang, F. Han, Q. Lu, and J. Qu, "Firing pattern and synchronization property analysis in a network model of the olfactory bulb," Cognitive Neurodynamics, vol. 6, no. 2, pp. 203209, 2012.

[14] T. Kreuz, J. S. Haas, A. Morelli, H. D. I. Abarbanel, and A. Politi, "Measuring spike train synchrony," Journal of Neuroscience Methods, vol. 165, no. 1, pp. 151-161, 2007. 


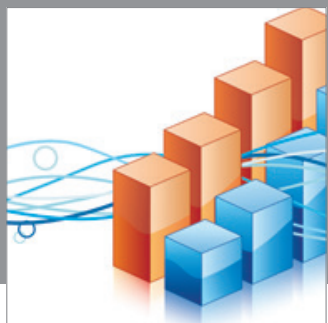

Advances in

Operations Research

mansans

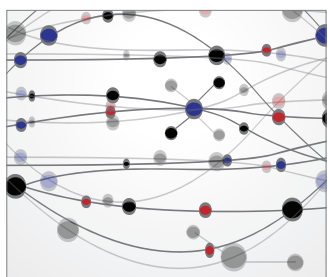

The Scientific World Journal
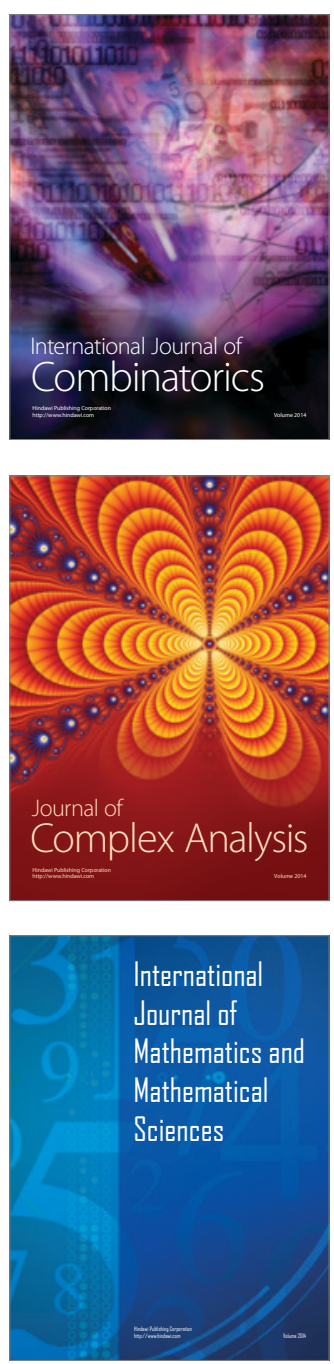
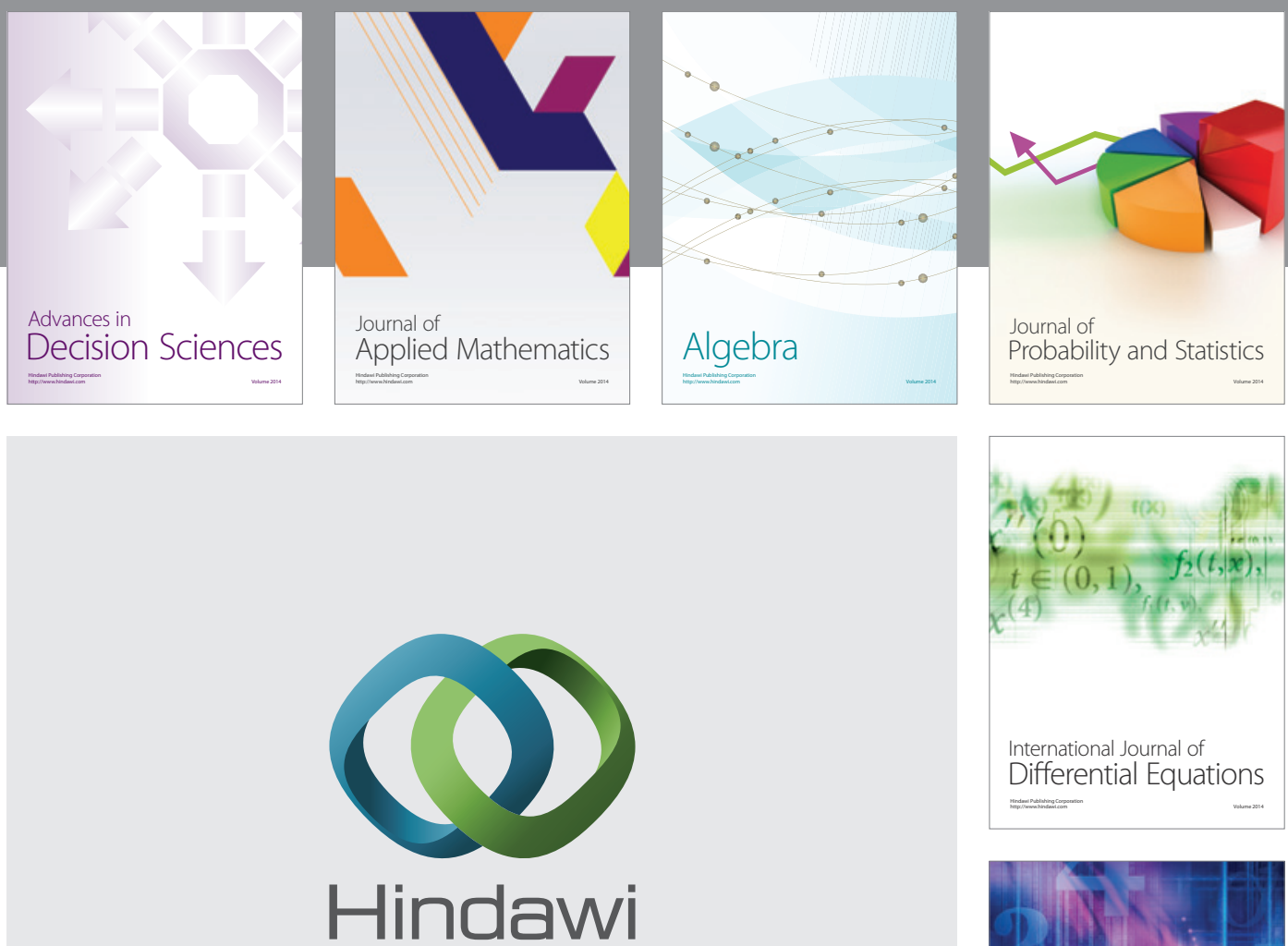

Submit your manuscripts at http://www.hindawi.com
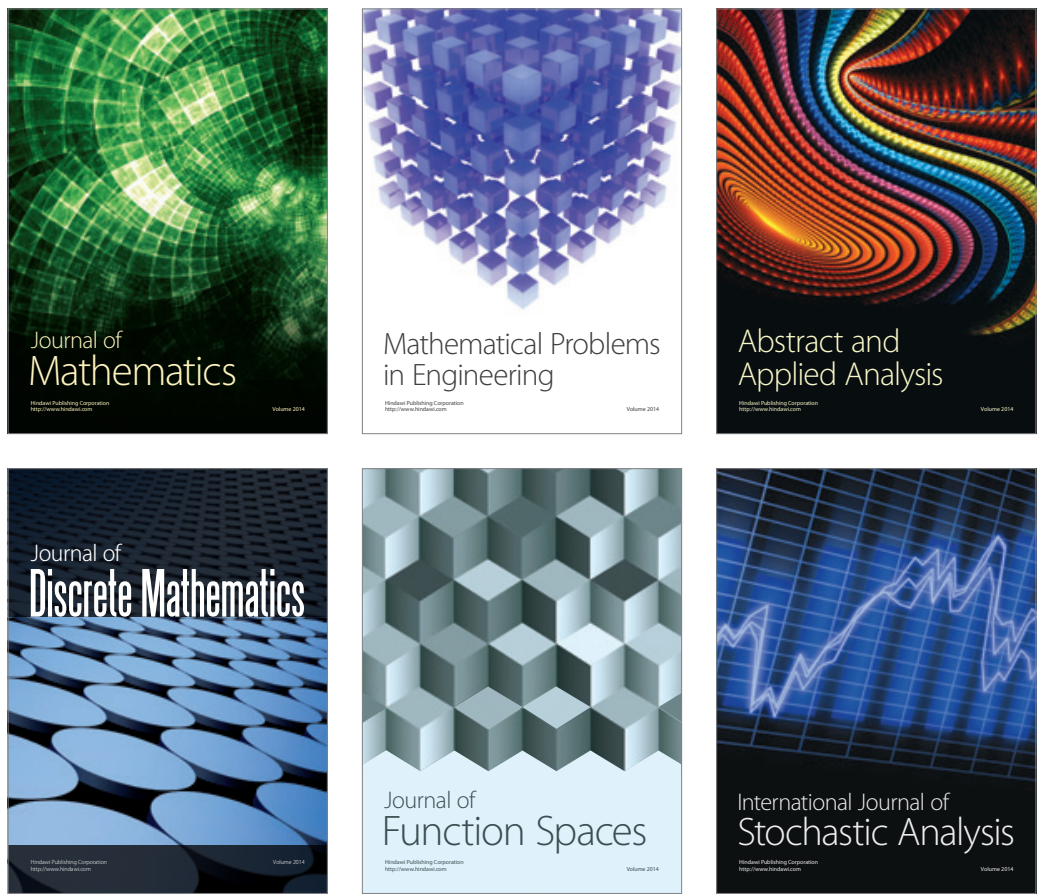

Journal of

Function Spaces

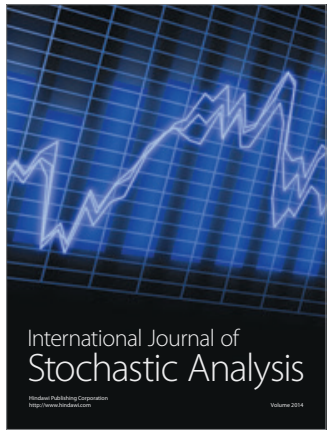

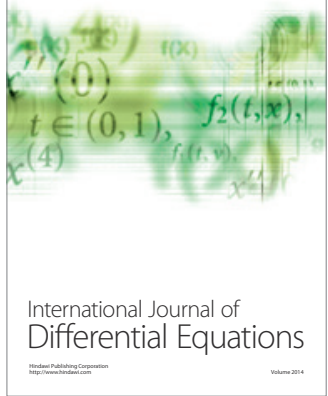
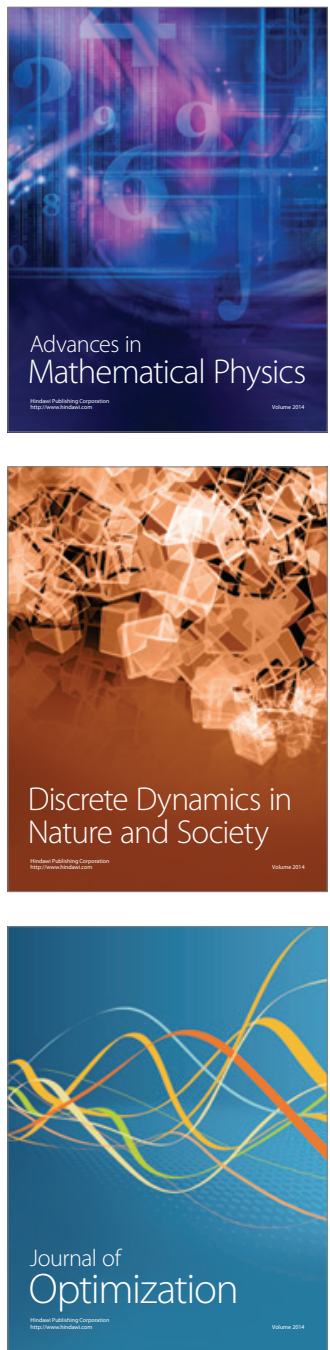